\title{
A biodiversity assessment of hard corals in dive spots within Dampier Straits Marine Protected Area in Raja Ampat, West Papua, Indonesia
}

\author{
YUANIKE ${ }^{1,2, \bullet}$, FREDINAN YULIANDA $^{1}$, DIETRIECH G. BENGEN ${ }^{3}$, ROKHMIN DAHURI $^{1}$, JEMMY SOUHOKA $^{4}$ \\ ${ }^{1}$ Coastal and Marine Resources Management Program, Faculty of Fisheries and Marine Science, Graduate School, Institut Pertanian Bogor. Jl. Raya \\ Dramaga, IPB Campus, Bogor 16680, West Java, Indonesia. "email: yuanike.kaber@ gmail.com \\ ${ }_{2}^{2}$ Marine Science Department, Faculty of Fisheries and Marine Science, Universitas Papua. J1. Gunung Salju, Amban, Manokwari 98314, West Papua, \\ Indonesia \\ ${ }^{3}$ Departement of Marine Science, Faculty of Fisheries and Marine Science, Institut Pertanian Bogor. Jl. Raya Dramaga, IPB Campus, Bogor 16680, West \\ Java, Indonesia \\ ${ }^{4}$ Integrated Services Unit for Marine Conservation of Bitung, Indonesian Institut of Sciences. J1. Tandurusa No. 1, Bitung 95511, North Sulawesi, \\ Indonesia
}

Manuscript received: 14 February 2019. Revision accepted: 29 March 2019.

\begin{abstract}
Yuanike, Yulianda F, Bengen DG, Dahuri R, Souhoka J. 2019. A biodiversity assessment of hard corals in dive spots within Dampier Straits Marine Protected Area in Raja Ampat, West Papua, Indonesia. Biodiversitas 20: 1198-1207. Hard coral dominates coral reef ecosystems and has important functions and interactions in communities of marine biota. The structure and diversity of hard coral communities is very important to quantify, especially for the management of coral reef ecosystems. The purpose of this research is to identify the diversity of hard coral types, and quantify the condition of hard coral and their distributions in 10 marine stations in Dampier Straits Marine Protected Areas, Raja Ampat. The research method used was line intercept transects measured at two depths, 3 $\mathrm{m}$ and $6 \mathrm{~m}$. The results showed there were 141 hard coral species included in 16 families. The percentage live cover of hard coral was in good conditions with an overall average coverage value of $64,24 \%$. The biodiversity index $(\mathrm{H})$ ranged from $0,84-1,23$, the evenness index of species ranged from $0,73-0,94$, and the species dominance index ranged from $0,05-0,25$, indicating a high biodiversity and a lack of dominance by a single species. Although cover was slightly higher at $3 \mathrm{~m}$ depth, diversity was slightly higher at $6 \mathrm{~m}$ depth. In general, the 10 research stations in Dampier Straits has hard coral in good condition and the diversity of hard coral species is very productive and has stable growth compared with other sites in Indonesia.
\end{abstract}

Keywords: Dampier Straits, hard coral, marine protected area, Raja Ampat, structure of community

\section{INTRODUCTION}

The waters of Indonesia are home to a very rich biodiversity of corals and reef fish with high economic value. The waters of West Papua's Bird's Head Seascape have the highest biodiversity compared with all other areas in Indonesia. Dampier Straits Marine Protected Area has an area of 366.000 hectares and is located among the Raja Ampat marine protected areas. Marine protected areas in Raja Ampat were promoted by the legacy of Raja Ampat Regency (Peraturan Bupati or government decree) No. 66 in 2007, the legacy in Raja Ampat Regency (Peraturan Daerah or local regulation) No. 27 in 2008 and the legacy of Raja Ampat Regency (Peraturan Bupati or government decree) No. 5 in 2009 (Boli et al. 2014; Mustaghfirin et al. 2012).

The water of Dampier Straits separates the islands of Batanta and Waigeo, and becomes one of the entrance gates of the masses of water in the Pacific Ocean that will flow into the Indian Ocean (Indonesian Throughflow). Dampier Straits water has strong currents and there are frequent occurrences of cold water upwelling phenomena. The resulting stirring of water masses brings together different temperatures, and brings rich nutrients to the surface that will be used by marine biotas, like coral, for the processes of reef formation. The resulting areas of reef formation consist of specific types of habitat leading to many kinds of reefs seascapes like fringing reefs and patch reefs.

Raja Ampat is an archipelagic regency and consists of three coastal and marine resources ecosystems: coral reefs, sea grass and mangroves. These ecosystems reveal the richness of natural resources in Raja Ampat, being very diverse and having important impacts on the economy and on human lifestyles. Specifically, the coral reef ecosystems produced by the main formations of hard corals play an important role in the function of the marine ecosystem, including their ecological interactions.

The hard corals are included in the Class Anthozoa, order Scleractinia, and live sessile lifestyles, meaning they are attached to the substrate. The hard coral is usually found living in shallow water, in less than $40 \mathrm{~m}$ depth (Nybakken and Bertness 2004). Sumadhiharga et al. (2006) stated that hard coral ecosystems are associated with other marine biota including fishes. The hard coral in Indonesia consists of 590 species in 80 genera (Suharsono 2010). The waters of Indonesia has coverages of $69.42 \%$ coral reef, including up to 850 species of coral, and corals have a high diversity of morphologies and functions (McWilliam et al. 2018). The research of coral reefs in Raja Ampat waters 
shows high level of biodiversity, composed of up to 553 species, representing $75 \%$ coral species in the world (Veron et al. 2009; Wallace et al. 2011 in Mustaghfirin et al. 2012). Suharsono (2010) stated that hard coral growth in Indonesia peaks around Sulawesi, Moluccas, Halmahera, Bali, West Nusa Tenggara, East Nusa Tenggara, Raja Ampat islands, the coasts of Papua Barat, Aru and Kei islands. Consequently, Raja Ampat is the centerpoint of the highest diversity of hard corals in the world (Veron et al. 2009; Allen and Erdmann 2009; Huffard et al. 2009; Mangubhai et al. 2012 in Mustaghfirin et al. 2012). However, the growth of infrastructure development in the coastal area and lack of good management in marine resources leads to degradation of the marine environment in Dampier Straits, and this can impact the hard coral diversity and growth in the ecosystem (Larsen et al. 2011). Consequently, it is important to quantify and monitor the biodiversity of corals in the area.

The the aims of the study is to identify the diversity of hard coral types, and quantify the condition of hard coral and their distributions in 10 marine stations in Dampier Straits Marine Protected Areas, Raja Ampat.

\section{MATERIALS AND METHODS}

\section{Study area}

The research assessment of hard coral in waters of Dampier Strait, Raja Ampat District, West Papua Province, Indonesia was conducted in April and November 2016. The data were collected from 10 dive spots or research stations. These were Five Rocks (Gam islands) (Station 1), Friwen Monda (Station 2), Mellisa Garden (Station 3), Lalosi Reef
(Station 4), Cape Kri (Station 5), Urun island (Station 6), Wai island (Station 7), Dayan island (Station 8), Cape Raspapir (Station 9) and coast waters of Yensawai (Station 10). The position of the stations is shown in Table 1 and the map of the research stations in Figure 1.

\section{Procedures}

The equipment for the collection of the data were scuba diving gear, underwater paper, motor boat, roll meters (100m), underwater camera and Global Positioning System (GPS). The assessment of hard coral and the component of coral reef ecosystem was conducted using the Line Intercept Transect (LIT) method (English et al. 1997) and were done in $3 \mathrm{~m}$ and $6 \mathrm{~m}$ depth for every research station. The observation of the coral reefs bentic component had been done by noting all of the components that pass by the meter rollers that is drawn along 10 meters and repeated three times.

Tabel 1. The position and location of hard coral assessment research stations in waters of Raja Ampat, 2016.

\begin{tabular}{lll}
\hline $\begin{array}{l}\text { No. } \\
\text { station }\end{array}$ & Position & Location \\
\hline 1 & $\mathrm{~S} 00^{\circ} 4510.7 ; \mathrm{E} 130^{\circ} 6973.3$ & Five Rocks \\
2 & $\mathrm{~S} 00^{\circ} 4777.5 ; \mathrm{E} 130^{\circ} 6963.2$ & Friwen Bonda \\
3 & $\mathrm{~S} 00^{\circ} 5896.7 ; \mathrm{E} 130^{\circ} 3159.3$ & Mellisa Garden \\
4 & $\mathrm{~S} 00^{\circ} 5482.5 ; \mathrm{E} 130^{\circ} 4963.8$ & Lalosi Reef \\
5 & $\mathrm{~S} 00^{\circ} 5575.4 ; \mathrm{E} 130^{\circ} 6893.5$ & Cape Kri \\
6 & $\mathrm{~S} 00^{\circ} 4637.8 ; \mathrm{E} 130^{\circ} 4049.5$ & Urun Island \\
7 & $\mathrm{~S} 00^{\circ} 4144.9 ; \mathrm{E} 130^{\circ} 4234.1$ & Wai Island \\
8 & $\mathrm{~S} 00^{\circ} 4812.8 ; \mathrm{E} 130^{\circ} 3900.8$ & Dayan Island \\
9 & $\mathrm{~S} 00^{\circ} 4800.3 ; \mathrm{E} 130^{\circ} 3854.2$ & Cape Raspapir \\
10 & $\mathrm{~S} 00^{\circ} 4836.4 ; \mathrm{E} 130^{\circ} 4124.3$ & Coast waters of Yensawai \\
\hline
\end{tabular}

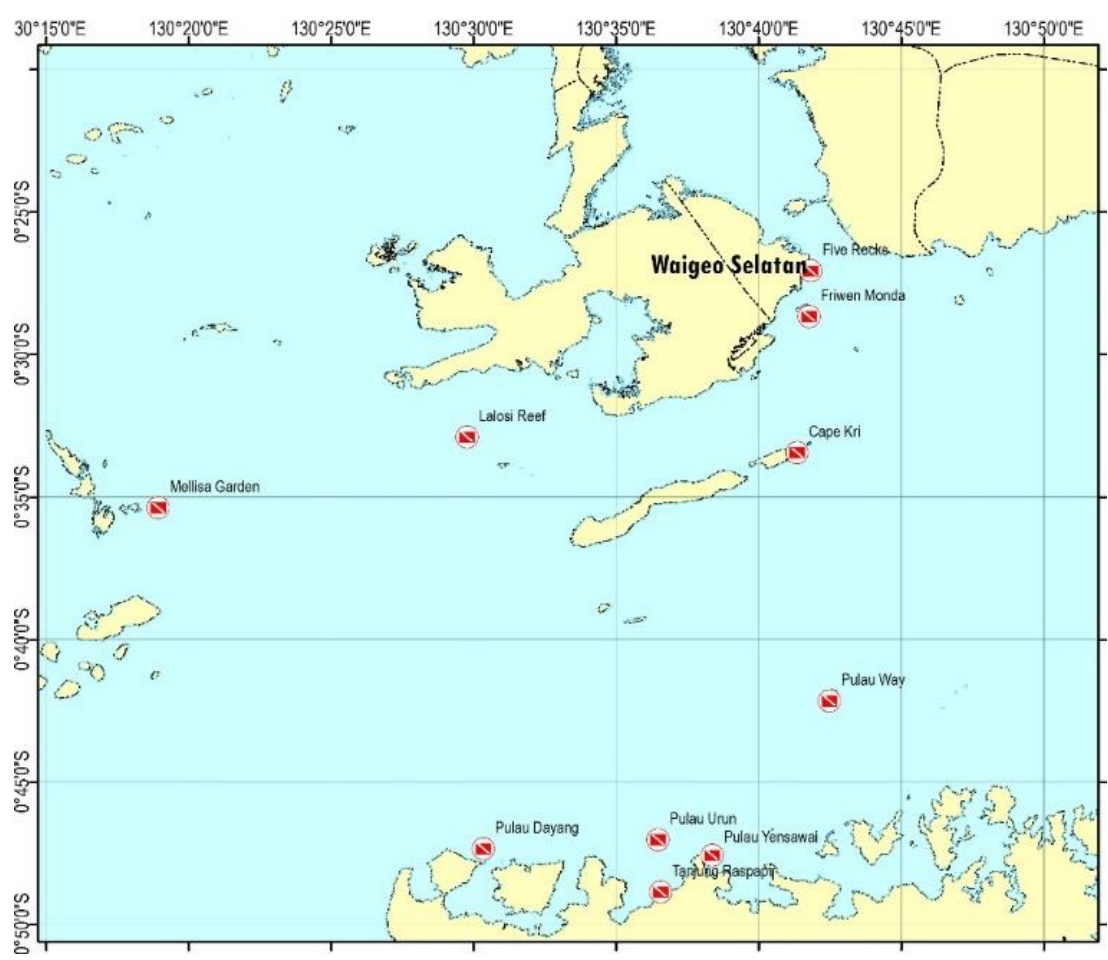

Figure 1. The map of research stations (rectangle in red circle) of hard coral assessment in Dampier Strait waters in Raja Ampat, West Papua, Indonesia, 2016 
The diver put out $70 \mathrm{~m}$ of the roll meter along the shoreline in $3 \mathrm{~m}$ and $5 \mathrm{~m}$ depth. The LIT data was collected in $0-10 \mathrm{~m}, 30-40 \mathrm{~m}$ dan $60-70 \mathrm{~m}$. All of the biota under the roll meter were noted to a detailed level every centimeter $(\mathrm{cm})$. The morphometric code of bentic components used was the prototcol developed by the Australian Institute of Marine Science (AIMS). The method used for coral reef site indentification is COREMAP-Institute of Sciences Indonesia phase I and II. The identification of hard coral was done in the field, but the unknown samples were collected and identified in the laboratory office in Integrated Services Unit for Marine Conservation-Bitung Institute of Sciences Indonesia based on the book of identification published by Veron (1986), Moll and Moka (1986), Huang and Mao (2012), Koh and Chou (1989), and Suharsono (2010).

\section{Data analysis}

The data from transects was analysed in hard coral percentage live cover using the Microsoft Excel program, and included the analysis of species biodiversity (biodiversity index) and species evenness (evenness index) with the application of Biodiversity Pro.4. The important value used was by species biodeviersity and species evenness, with a number of individuals (colonies) of every species of hard coral. The analyzed live cover percentage $(\%)$, species biodiversity $(\mathrm{H})$ and species evenness $(\mathrm{j})$ has been done following the Odum formulae (1971):

The percentage of hard coral life form:

$$
\% \text { life form }=\frac{\text { Length of every colony }}{\text { Length of transect }} \times 100
$$

Species biodiversity $(\mathrm{H})$ :

$\mathrm{H}=-\sum(\mathrm{ni} / \mathrm{N}) \log (\mathrm{ni} / \mathrm{N})$

Species evenness $(\mathrm{j})$ :

$\mathrm{j}=\mathrm{H} / \log \mathrm{S}$

Species dominancy $(\mathrm{H})$ :

$\mathrm{D}=(\mathrm{ni} / \mathrm{N})^{2}$

Where:

$\mathrm{H}$ : a value of species biodiversity

$\mathrm{Ni}$ : number of individuals

$\mathrm{N}$ : a total of individuals

$\mathrm{j}$ : a value of species evenness

S: a total number of species

D: value of species dominance

\section{RESULTS AND DISCUSSION}

\section{Biodiversity of coral reefs}

The Dampier Straits research stations monitored in this study had characteristics of a coastline that is dominated by coastal plants, mangroves, rock, and sand. The distance of the coral reef assessment was varied from $3 \mathrm{~m}$ (Station 5) until $50 \mathrm{~m}$ (Station 10). Station 2, 3 and station 4 were located on an atoll in the center of the waters. The substrate of bottom waters in research stations consists of sand, rubble, and rock. In the analysis of hard corals at the research stations, the number of species found was 141 species and these were included in 16 families (Table 2). The highest species richness was found in Station 6 (77 species included in 15 families), and the lowest was found in Station 10 (49 species that represented 14 families) (Table 2).

\section{The percentage of hard coral life form}

The highest percentage of live hard coral cover in $3 \mathrm{~m}$ depth, Dampier Strait waters was found at Station 5 $(86.83 \%)$ and the lowest at Station $8(44.17 \%)$ (Table 3). The highest percentage of live hard coral cover in $6 \mathrm{~m}$ depth was found at Station $3(87.40 \%)$ and the lowest at Station $2(51.17 \%)$ (Table 4$)$.

\section{The value of hard coral diversity, evenness and dominance index}

The value of hard coral diversity index $(\mathrm{H})$, evenness index (j) and dominance index (D) in $3 \mathrm{~m}$ and $6 \mathrm{~m}$ depth in Dampier Strait waters research station are listed in Table 5.

Table 2. The composition of hard coral species in Dampier Strait

\begin{tabular}{|c|c|c|c|c|c|c|c|c|c|c|}
\hline \multirow{2}{*}{ Family/species } & \multicolumn{10}{|c|}{ Research station } \\
\hline & 1 & 2 & 3 & 4 & 5 & 6 & 7 & 8 & 9 & 10 \\
\hline \multicolumn{11}{|l|}{ I. POCILLOPORIDAE } \\
\hline Pocilopora damicornis & + & - & + & - & + & - & - & - & - & - \\
\hline P. verrucosa & + & + & + & + & + & + & + & + & + & - \\
\hline Seriotophora caliendrum & + & + & + & + & + & + & + & + & + & + \\
\hline S. hystrix & + & + & + & - & + & + & + & + & + & + \\
\hline Stylophora pistillata & + & + & + & + & + & + & + & - & + & + \\
\hline \multicolumn{11}{|l|}{ II. ACROPORIDAE } \\
\hline Montipora efflorescens & - & - & - & - & + & - & - & - & - & - \\
\hline M. danae & - & + & + & - & - & + & + & + & - & - \\
\hline M. foliosa & - & + & + & + & + & + & - & + & + & + \\
\hline M. hoffmeisteri & - & - & + & - & + & + & + & + & + & + \\
\hline M. informis & + & + & + & + & + & + & - & + & + & - \\
\hline M. monasteriata & - & - & + & - & + & - & - & - & + & - \\
\hline M. spongodes & - & - & + & - & + & - & - & - & - & - \\
\hline M. undata & - & - & + & + & - & + & + & + & + & - \\
\hline Montipora sp. & + & + & - & + & - & - & - & - & - & + \\
\hline Acropora acuminata & + & - & + & - & + & - & - & - & - & - \\
\hline A. aspera & + & - & - & - & + & + & - & - & - & - \\
\hline A.cerealis & - & + & + & - & - & - & - & - & - & - \\
\hline A.clathrata & + & + & + & + & + & + & + & + & + & - \\
\hline A. cytherea & - & - & + & + & + & - & + & - & + & - \\
\hline A. dendrum & + & - & + & + & + & - & - & - & - & - \\
\hline A. digitifera & + & - & - & - & - & - & - & - & - & - \\
\hline A. donei & - & - & + & + & - & + & - & - & + & - \\
\hline A. echinata & - & - & - & - & - & - & - & - & + & + \\
\hline A. florida & + & + & + & + & - & + & + & - & + & - \\
\hline A. Formosa & + & + & - & + & + & + & + & + & + & + \\
\hline A. gemmifera & - & - & - & - & - & + & + & - & - & - \\
\hline A. granulosa & + & - & - & - & - & - & - & - & - & - \\
\hline A. grandis & - & - & - & - & + & - & - & - & - & - \\
\hline A. horrida & - & - & - & - & - & - & - & - & + & - \\
\hline A. humilis & - & + & - & - & - & + & - & - & - & - \\
\hline
\end{tabular}
waters, Raja Ampat, 2016 
A. hyacinthus

A. intermedia

A. loripes

A. millepora

A. nobilis

A.nasuta

A. palifera

A. pulchra

A. samoensis

A. subglabra

A. tenuis

A. valenciennesi

A. yongei

Acropora sp.

Anacropora puartogalerae

Astreopora myriophthalma

A. gracillis

III. PORITIDAE

Porites cylindrical

P. lichen

P. lobate

P. lutea

P. nigrecens

P. mayeri

P. rus

Goniopora columna

G. lobate

Goniopora sp.

Alveopora catalai

IV. SIDERASTREIDAE

Psammocora digitata

P. superficialis

Coscinaraea columna

V. AGARICIIDAE

Pavona cactus

P. decussate

$P$. varians

Coeloseris mayeri

Leptoseris explanata

Leptoseris sp.

Gardineroseris planulata

Pachyseris speciosa

$P$. rugose

VI. FUNGIIDAE

Fungia concinna

F. danai

F. fungites

F. horrida

F. paumotensis

F. repanda

F. scutaria

Ctnactis echinata

Heliofungia actiniformis

Herpolitha limax

Halomitra pileus

Polyphyllia talpina

VII. OCULINIDAE

Galaxea astreata

G. fascicularis

VIII. PECTINIDAE

Oxypora glabra

O. lacera

Mycedium elephantotus

Pectinia lactuca

P. paeonia

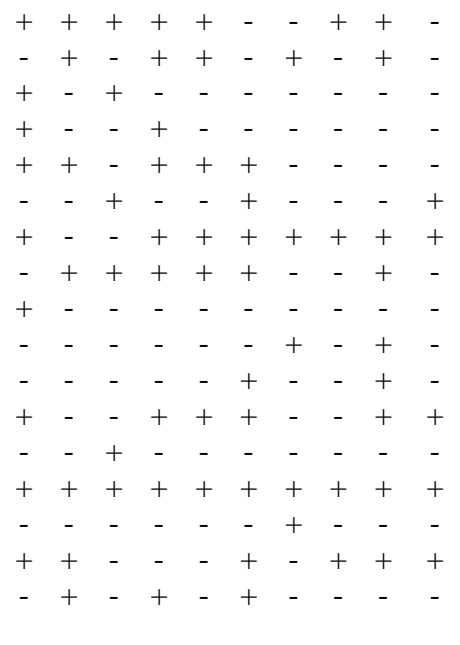

$+\quad-\quad-\quad-\quad+\quad+\quad+\quad+$

$\begin{array}{llllllllll}- & - & - & + & - & - & + & - & - & - \\ + & + & + & + & + & + & + & + & + & +\end{array}$

$+++++++++$

$+-\quad+\quad++-\quad-$

$-\quad-\quad-\quad-\quad-\quad-$

$\begin{array}{cccccccccc}- & - & - & - & - & + & - & - & + & + \\ - & + & + & + & - & + & + & + & + & +\end{array}$

$-\quad-\quad-\quad+\quad-\quad-\quad-$
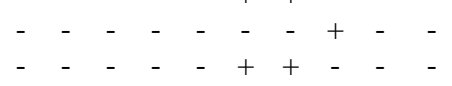

P. teres

IX. MUSSIDAE

Acanthastrea hillae

A. lordhowensis

A. hemprichii

Lobophyllia hemprichii

Symphyllia recta

$S$. radians

S. wilsoni

Symphyllia sp.

X. MERULINIDAE

Hydnophora exesa

H. rigida

Merulina ampliata

M. scabricula

Scapophyllia cylindrica

XI. ASTROCOENIDAE

Stylocoeniella armata

Palaustrea ramosa

XII. FAVIIDAE

Favites abdita

$F$. complanata

F. halicora

Favites sp.

Favia maritima

F. matthaii

$F$. stelligera

F. danae

F. pallida

Favia sp.

Goniastrea aspera

G. favulus

G. pectinate

G. retiformis

Goniastrea sp.

Platygyra daedalae

Leptoria phrygia

Oulophyllia bennettae

Montastrea annuligera

M. curta

Diploastrea heliopora

Cyphastrea microphthalma

C. chalcidicum

Echinopora lamellosa

Echinopora sp.

XIII. CARYOPHYLLIDAE

Euphyllia ancora

E. glabrescens

Plerogyra sinuosa

Physogyra lichtensteini

XIV.DENDROPHYLLIDAE

Turbinaria mesenterina

T. reniformis

T. frondens

Tubastrea micrantha

T. faulkneri

XV. MILLEPORIDAE

Millepora sp.

XVI. HELIOPHORIDAE

Heliophora coerulea

Species

Family

$\begin{array}{llllllllll}52 & 56 & 57 & 62 & 50 & 77 & 61 & 51 & 74 & 49\end{array}$

$\begin{array}{llllllllll}12 & 13 & 13 & 14 & 12 & 15 & 15 & 15 & 16 & 14\end{array}$ 
Table 3. The percentage of hard coral life form, biotic and abiotic component of hard coral (3 m depth) in Dampier Strait waters, Raja Ampat, 2016

\begin{tabular}{|c|c|c|c|c|c|c|c|c|c|c|}
\hline \multirow{2}{*}{ Benthic components } & \multicolumn{10}{|c|}{ Research station } \\
\hline & $\mathbf{1}$ & 2 & 3 & 4 & 5 & 6 & 7 & 8 & 9 & 10 \\
\hline Life coral (LC) & 49.70 & 52.50 & 85.33 & 64.67 & 86.83 & 56.31 & 55.97 & 44.17 & 79.10 & 81.30 \\
\hline Soft Coral (SC) & 17.90 & 35.33 & 5.33 & 20.67 & 10.17 & 3.00 & 21.93 & 40.20 & 16.23 & 0.00 \\
\hline Sponges (Sp) & 0.83 & 0.33 & 1.00 & 0.67 & 0.00 & 3.63 & 0.00 & 0.67 & 1.33 & 0.00 \\
\hline Macro algae (MA) & 1.67 & 0.00 & 0.00 & 0.00 & 0.00 & 5.56 & 0.00 & 0.00 & 0.67 & 2.93 \\
\hline Halimeda (HA) & 0.00 & 0.00 & 0.00 & 0.00 & 0.00 & 0.00 & 0.00 & 0.00 & 0.00 & 0.00 \\
\hline Others (OT) & 0.00 & 1.33 & 0.00 & 0.00 & 0.00 & 1.53 & 0.00 & 0.00 & 0.00 & 0.00 \\
\hline Rubble (R) & 5.67 & 1.67 & 2.00 & 1.83 & 0.00 & 9.33 & 19.70 & 8.83 & 1.33 & 14.77 \\
\hline Sand $(\mathrm{S})$ & 4.43 & 2.00 & 0.00 & 0.00 & 0.00 & 10.33 & 1.10 & 6.13 & 1.33 & 0.00 \\
\hline Silt ( Si) & 0.00 & 0.00 & 0.00 & 0.00 & 0.00 & 0.00 & 0.00 & 0.00 & 0.00 & 0.00 \\
\hline Dead coral with algae (DCA) & 19.80 & 6.83 & 6.33 & 12.17 & 3.00 & 10.30 & 1.30 & 0.00 & 0.00 & 1.00 \\
\hline Dead coral (DC) & 0.00 & 0.00 & 0.00 & 0.00 & 0.00 & 0.00 & 0.00 & 0.00 & 0.00 & 0.00 \\
\hline Rock (RCK) & 0.00 & 0.00 & 0.00 & 0.00 & 0.00 & 0.00 & 0.00 & 0.00 & 0.00 & 0.00 \\
\hline
\end{tabular}

Table 4. The percentage of hard coral life form, biotic and abiotic component of hard coral (6 m depth) in Dampier Strait waters, Raja Ampat, 2016.

\begin{tabular}{|c|c|c|c|c|c|c|c|c|c|c|}
\hline \multirow{2}{*}{ Benthic components } & \multicolumn{10}{|c|}{ Research station } \\
\hline & 1 & 2 & 3 & 4 & 5 & 6 & 7 & 8 & 9 & 10 \\
\hline Life coral (LC) & 60.97 & 51.17 & 87.40 & 52.33 & 52.17 & 55.93 & 63.67 & 66.33 & 58.70 & 80.27 \\
\hline Soft Coral (SC) & 3.83 & 27.67 & 6.00 & 29.17 & 21.50 & 0.33 & 14.00 & 25.07 & 8.10 & 0.00 \\
\hline Sponges (Sp) & 0.00 & 2.07 & 0.00 & 2.33 & 0.00 & 0.00 & 1.37 & 1.50 & 1.00 & 0.00 \\
\hline Macro algae (MA) & 0.67 & 1.17 & 0.00 & 0.00 & 0.00 & 2.67 & 0.00 & 0.00 & 4.33 & 0.00 \\
\hline Halimeda (HA) & 0.00 & 0.00 & 0.00 & 0.00 & 0.00 & 0.00 & 0.00 & 0.00 & 0.00 & 0.00 \\
\hline Others (OT) & 0.00 & 1.00 & 3.50 & 0.00 & 0.00 & 1.00 & 0.00 & 0.00 & 0.00 & 0.00 \\
\hline Rubble (R) & 23.03 & 0.83 & 1.33 & 5.67 & 19.33 & 28.03 & 16.47 & 1.33 & 23.53 & 19.07 \\
\hline Sand (S) & 4.00 & 4.20 & 0.00 & 2.00 & 3.67 & 4.67 & 3.50 & 5.10 & 4.33 & 0.00 \\
\hline Silt ( Si) & 0.00 & 0.00 & 0.00 & 0.00 & 0.00 & 0.00 & 0.00 & 0.00 & 0.00 & 0.00 \\
\hline Dead coral with algae (DCA) & 7.50 & 11.90 & 1.77 & 8.50 & 3.33 & 7.37 & 1.00 & 0.67 & 0.00 & 0.67 \\
\hline Dead coral (DC) & 0.00 & 0.00 & 0.00 & 0.00 & 0.00 & 0.00 & 0.00 & 0.00 & 0.00 & 0.00 \\
\hline Rock (RCK) & 0.00 & 0.00 & 0.00 & 0.00 & 0.00 & 0.00 & 0.00 & 0.00 & 0.00 & 0.00 \\
\hline
\end{tabular}

Table 5. The value of hard coral diversity index $(\mathrm{H})$, evenness index (j) and dominance index (D) in $3 \mathrm{~m}$ and $6 \mathrm{~m}$ depth in Dampier Strait waters research station, 2016.

\begin{tabular}{|c|c|c|c|c|c|c|c|c|c|c|c|}
\hline \multirow{2}{*}{ Depth } & \multirow{2}{*}{ Components } & \multicolumn{10}{|c|}{ Research station } \\
\hline & & 1 & 2 & 3 & 4 & 5 & 6 & 7 & 8 & 9 & 10 \\
\hline \multirow[t]{5}{*}{$3 \mathrm{~m}$} & Number of species & 14 & 20 & 17 & 22 & 19 & 14 & 17 & 9 & 20 & 18 \\
\hline & Number of individual & 50 & 52 & 60 & 58 & 47 & 59 & 50 & 23 & 61 & 80 \\
\hline & Diversity index $(\mathrm{H})$ & 0.99 & 1.13 & 1.00 & 1.23 & 1.12 & 1.00 & 1.09 & 0.84 & 1.06 & 0.93 \\
\hline & Evenness index (j) & 0.87 & 0.87 & 0.81 & 0.91 & 0.88 & 0.87 & 0.89 & 0.88 & 0.82 & 0.74 \\
\hline & Dominancy index (D) & 0.13 & 0.10 & 0.17 & 0.07 & 0.10 & 0.12 & 0.10 & 0.18 & 0.15 & 0.19 \\
\hline \multirow[t]{5}{*}{$6 \mathrm{~m}$} & Number of species & 16 & 31 & 16 & 19 & 19 & 16 & 21 & 15 & 19 & 11 \\
\hline & Number of individual & 46 & 58 & 50 & 46 & 55 & 59 & 45 & 41 & 45 & 45 \\
\hline & Diversity index $(\mathrm{H})$ & 1.07 & 1.39 & 1.09 & 1.20 & 1.16 & 1.03 & 1.13 & 1.05 & 1.13 & 0.76 \\
\hline & Evenness index (j) & 0.89 & 0.93 & 0.91 & 0.94 & 0.91 & 0.85 & 0.85 & 0.89 & 0.88 & 0.73 \\
\hline & Dominancy index (D) & 0.11 & 0.05 & 0.10 & 0.07 & 0.09 & 0.12 & 0.13 & 0.11 & 0.11 & 0.25 \\
\hline
\end{tabular}

\section{The length measurement of coral reef colony}

The length measurement of coral reef colony in $3 \mathrm{~m}$ and $6 \mathrm{~m}$ depth in Dampier Strait waters research station are listed in Table 6, 7 and 8.

\section{Discussion}

Biodiversity of coral reefs

The identification of hard coral in $3 \mathrm{~m}$ and $6 \mathrm{~m}$ revealed 141 species of hard coral (Table 2). The total number of 
Table 6. The total length of hard coral colonies, presence, and frequency of presence every hard coral species in $3 \mathrm{~m}$ depth, Dampier Strait waters research station, 2016.

\begin{tabular}{|c|c|c|c|}
\hline Species of hard coral & $\begin{array}{c}\text { Total length of } \\
\text { colonies } \\
\text { (\% coverage) }\end{array}$ & Presence & $\begin{array}{c}\text { Freq. of } \\
\text { presence } \\
(\%)\end{array}$ \\
\hline Acropora sp. & 21.32 & 10 & 100 \\
\hline Porites lutea & 9.17 & 9 & 90 \\
\hline Echinopora lamellosa & 6.34 & 1 & 10 \\
\hline Seriatophora hystrix & 4.15 & 5 & 50 \\
\hline Acropora clathrata & 3.75 & 5 & 50 \\
\hline Porites nigrecens & 3.75 & 5 & 50 \\
\hline Seriatophora caliendrum & 3.72 & 8 & 80 \\
\hline Montipora informis & 3.61 & 5 & 50 \\
\hline Stylophora pistillata & 3.55 & 7 & 70 \\
\hline Millepora sp. & 3.28 & 2 & 20 \\
\hline Goniopora columna & 2.79 & 3 & 30 \\
\hline Acropora hyacinthus & 2.67 & 5 & 50 \\
\hline Acropora palifera & 2.28 & 4 & 40 \\
\hline Pocillopora verrucossa & 2.20 & 6 & 60 \\
\hline Acropora formosa & 2.08 & 4 & 40 \\
\hline Montipora foliosa & 2.06 & 3 & 30 \\
\hline Favites sp. & 1.56 & 6 & 60 \\
\hline Acropora florida & 1.52 & 5 & 50 \\
\hline Acropora pulchra & 1.37 & 5 & 50 \\
\hline Acropora valleciennesi & 1.37 & 1 & 10 \\
\hline Pectinia lactuca & 1.27 & 3 & 30 \\
\hline Porites cylindrica & 1.26 & 2 & 20 \\
\hline Hydnophora rigida & 1.25 & 5 & 50 \\
\hline Galaxea astreata & 1.21 & 4 & 40 \\
\hline Acropora aspera & 1.07 & 2 & 20 \\
\hline Fungia concinna & 1.02 & 6 & 60 \\
\hline Porites lobata & 1.02 & 3 & 30 \\
\hline Fungia fungites & 0.68 & 4 & 40 \\
\hline Montipora undata & 0.64 & 2 & 20 \\
\hline Merulina ampliata & 0.62 & 2 & 20 \\
\hline Favites halicora & 0.56 & 3 & 30 \\
\hline Tubastrea micrantha & 0.56 & 1 & 10 \\
\hline Favia sp. & 0.46 & 2 & 20 \\
\hline Acropora nasuta & 0.42 & 1 & 10 \\
\hline Acropora grandis & 0.41 & 1 & 10 \\
\hline Montipora danae & 0.41 & 1 & 10 \\
\hline Oxypora lacera & 0.41 & 2 & 20 \\
\hline Montipora sp. & 0.38 & 3 & 30 \\
\hline Cyphastrea chalcidicum & 0.36 & 1 & 10 \\
\hline Diploastrea heliopora & 0.36 & 1 & 10 \\
\hline Goniastrea pectinata & 0.36 & 1 & 10 \\
\hline Pachyseris rugosa & 0.36 & 2 & 20 \\
\hline Symphyllia recta & 0.30 & 1 & 10 \\
\hline Heliofungia actiniformis & 0.30 & 2 & 20 \\
\hline Favites complanata & 0.25 & 2 & 20 \\
\hline Astreopora gracillis & 0.23 & 1 & 10 \\
\hline Acropora millepora & 0.20 & 1 & 10 \\
\hline Acropora nobillis & 0.20 & 2 & 20 \\
\hline Herpolitha limax & 0.20 & 2 & 20 \\
\hline Goniastrea sp. & 0.15 & 1 & 10 \\
\hline Palaustrea ramosa & 0.12 & 1 & 10 \\
\hline Echinopora sp. & 0.10 & 1 & 10 \\
\hline Halomitra pileus & 0.10 & 1 & 10 \\
\hline Goniastrea retiformis & 0.10 & 1 & 10 \\
\hline Acropora echinata & 0.06 & 1 & 10 \\
\hline Acropora intermedia & 0.05 & 1 & 10 \\
\hline Porites lichen & 0.05 & 1 & 10 \\
\hline The total of length & 100.00 & & \\
\hline
\end{tabular}

Table 7. The total length of hard coral colonies, presence, and frequency of presence every hard coral species in $6 \mathrm{~m}$ depth, Dampier Strait waters research station, 2016.

\begin{tabular}{|c|c|c|c|}
\hline Hard coral species & $\begin{array}{c}\text { Total length of } \\
\text { colonies } \\
\text { (\% coverage) }\end{array}$ & Presence & $\begin{array}{l}\text { Freq. of } \\
\text { presence } \\
(\%)\end{array}$ \\
\hline Acropora sp. & 14.15 & 8 & 80 \\
\hline Porites lutea & 9.06 & 8 & 80 \\
\hline Echinopora lamellosa & 7.61 & 1 & 10 \\
\hline Seriatophora hystrix & 4.67 & 7 & 70 \\
\hline Porites nigrecens & 4.19 & 7 & 70 \\
\hline Seriatophora caliendrum & 4.11 & 7 & 70 \\
\hline Montipora informis & 3.86 & 6 & 60 \\
\hline Acropora hyacinthus & 3.82 & 4 & 40 \\
\hline Montipora foliosa & 3.68 & 6 & 60 \\
\hline Stylophora pistillata & 3.19 & 7 & 70 \\
\hline Goniopora sp. & 2.49 & 1 & 10 \\
\hline Acropora valleciennesi & 2.44 & 3 & 30 \\
\hline Porites cylindrica & 2.18 & 3 & 30 \\
\hline Pocillopora verrucossa & 1.88 & 4 & 40 \\
\hline Acropora palifera & 1.84 & 4 & 40 \\
\hline Montipora danae & 1.70 & 2 & 20 \\
\hline Acropora clathrata & 1.53 & 6 & 60 \\
\hline Merulina ampliata & 1.43 & 3 & 30 \\
\hline Millepora sp. & 1.40 & 2 & 20 \\
\hline Favites sp. & 1.38 & 4 & 40 \\
\hline Fungia concinna & 1.38 & 8 & 80 \\
\hline Acropora formosa & 1.27 & 4 & 40 \\
\hline Acropora echinata & 1.25 & 1 & 10 \\
\hline Echinopora sp. & 1.25 & 3 & 30 \\
\hline Oxypora lacera & 1.18 & 1 & 10 \\
\hline Galaxea astreata & 1.18 & 5 & 50 \\
\hline Goniopora columna & 1.17 & 3 & 30 \\
\hline Diploastrea heliopora & 1.01 & 3 & 30 \\
\hline Montipora sp. & 0.87 & 2 & 20 \\
\hline Montipora undata & 0.85 & 5 & 50 \\
\hline Pectinia lactuca & 0.85 & 4 & 40 \\
\hline Porites rus & 0.85 & 2 & 20 \\
\hline Porites lobata & 0.75 & 3 & 30 \\
\hline Acropora aspera & 0.74 & 1 & 10 \\
\hline Astreopora gracillis & 0.74 & 2 & 20 \\
\hline Pachyseris rugosa & 0.69 & 3 & 30 \\
\hline Acropora horrida & 0.53 & 1 & 10 \\
\hline Acropora intermedia & 0.48 & 1 & 10 \\
\hline Acropora millepora & 0.45 & 1 & 10 \\
\hline Acropora nasuta & 0.42 & 2 & 20 \\
\hline Favia matthaii & 0.42 & 1 & 10 \\
\hline Acropora digitifera & 0.37 & 1 & 10 \\
\hline Acropora nobillis & 0.37 & 2 & 20 \\
\hline Acropora florida & 0.34 & 2 & 20 \\
\hline Fungia fungites & 0.32 & 2 & 20 \\
\hline Pavona decussatta & 0.32 & 1 & 10 \\
\hline Anacropora puartogalerae & 0.29 & 1 & 10 \\
\hline Acropora grandis & 0.26 & 1 & 10 \\
\hline Goniastrea pectinata & 0.26 & 2 & 20 \\
\hline Lobophyllia hemprichii & 0.26 & 2 & 20 \\
\hline Favites complanata & 0.21 & 2 & 20 \\
\hline Herpolitha limax & 0.21 & 2 & 20 \\
\hline Hydnophora rigida & 0.21 & 2 & 20 \\
\hline Leptoseris sp. & 0.21 & 1 & 10 \\
\hline Symphyllia sp. & 0.21 & 1 & 10 \\
\hline Acropora yongei & 0.16 & 1 & 10 \\
\hline Favites abdita & 0.16 & 1 & 10 \\
\hline Pavona teres & 0.16 & 1 & 10 \\
\hline Tubastrea micrantha & 0.16 & 1 & 10 \\
\hline Cyphastrea chalcidicum & 0.12 & 1 & 10 \\
\hline Acropora pulchra & 0.11 & 2 & 20 \\
\hline Euphyllia ancora & 0.11 & 1 & 10 \\
\hline Heliofungia actiniformis & 0.11 & 1 & 10 \\
\hline Coscinaraea sp. & 0.08 & 1 & 10 \\
\hline Acropora humillis & 0.05 & 1 & 10 \\
\hline Total of length & 100.00 & & \\
\hline
\end{tabular}


Table 8. The total length of hard coral colonies of 10 species which dominates (3m and 6m depth), in Dampier Strait marine protected area research station, 2016

\begin{tabular}{|c|c|c|c|c|c|c|c|c|c|c|c|c|}
\hline \multirow{2}{*}{ Depth } & \multirow{2}{*}{ Species } & \multicolumn{11}{|c|}{ Total length of colonies ( $\%$ coverage) } \\
\hline & & 1 & 2 & 3 & 4 & $5^{\circ}$ & 6 & 7 & 8 & 9 & 10 & Total \\
\hline \multirow[t]{10}{*}{$3 \mathrm{~m}$} & Acropora sp. & 4.77 & 1.31 & 4.53 & 4.77 & 15.50 & 4.91 & 12.40 & 18.84 & 30.00 & 2.98 & 100.00 \\
\hline & Porites lutea & 24.33 & 9.70 & 28.27 & 17.18 & 0.55 & 9.42 & 3.05 & 4.43 & 3.05 & 0.00 & 100.00 \\
\hline & Echinopora lamellosa & 0.00 & 0.00 & 0.00 & 0.00 & 0.00 & 0.00 & 0.00 & 0.00 & 0.00 & 100.00 & 100.00 \\
\hline & Seriatophora hystrix & 0.00 & 28.19 & 9.80 & 0.00 & 2.45 & 25.74 & 0.00 & 0.00 & 0.00 & 33.82 & 100.00 \\
\hline & Acropora clathrata & 4.34 & 0.00 & 17.62 & 25.07 & 48.78 & 0.00 & 0.00 & 0.00 & 4.20 & 0.00 & 100.00 \\
\hline & Porites nigrecens & 0.03 & 0.00 & 0.00 & 0.00 & 0.58 & 0.16 & 0.12 & 0.00 & 0.00 & 0.09 & 100.00 \\
\hline & Seriatophora caliendrum & 21.89 & 31.46 & 5.47 & 4.10 & 0.00 & 27.36 & 3.56 & 0.00 & 4.79 & 1.37 & 100.00 \\
\hline & Montipora informis & 4.93 & 22.54 & 46.48 & 21.83 & 4.23 & 0.00 & 0.00 & 0.00 & 0.00 & 0.00 & 100.00 \\
\hline & Stylophora pistillata & 7.88 & 0.00 & 14.33 & 0.00 & 14.33 & 2.87 & 17.05 & 0.00 & 37.11 & 6.45 & 100.00 \\
\hline & Millepora sp. & 0.00 & 0.00 & 0.00 & 0.00 & 27.09 & 72.91 & 0.00 & 0.00 & 0.00 & 0.00 & 100.00 \\
\hline \multirow[t]{10}{*}{$6 \mathrm{~m}$} & Acropora sp. & 0.00 & 2.51 & 6.37 & 4.61 & 9.74 & 1.87 & 38.58 & 13.11 & 23.22 & 0.00 & 100.00 \\
\hline & Porites lutea & 11.40 & 8.36 & 30.23 & 7.02 & 14.62 & 2.34 & 0.00 & 24.56 & 1.46 & 0.00 & 100.00 \\
\hline & Echinopora lamellosa & 0.00 & 0.00 & 0.00 & 0.00 & 0.00 & 0.00 & 0.00 & 0.00 & 0.00 & 100.00 & 100.00 \\
\hline & Seriatophora hystrix & 0.00 & 7.37 & 17.01 & 0.00 & 9.64 & 22.00 & 0.91 & 0.00 & 2.27 & 40.82 & 100.00 \\
\hline & Porites nigrecens & 48.10 & 0.00 & 0.00 & 7.59 & 3.16 & 29.75 & 3.80 & 2.53 & 5.06 & 0.00 & 100.00 \\
\hline & Seriatophora caliendrum & 27.06 & 6.44 & 15.46 & 0.00 & 15.46 & 29.64 & 3.35 & 0.00 & 2.58 & 0.00 & 100.00 \\
\hline & Montipora informis & 8.93 & 25.82 & 28.85 & 12.36 & 13.05 & 0.00 & 10.99 & 0.00 & 0.00 & 0.00 & 100.00 \\
\hline & Acropora hyacinthus & 26.39 & 0.00 & 47.92 & 21.53 & 0.00 & 0.00 & 0.00 & 0.00 & 4.17 & 0.00 & 100.00 \\
\hline & Montipora foliosa & 0.00 & 6.34 & 53.31 & 0.00 & 9.80 & 0.00 & 3.46 & 22.77 & 4.32 & 0.00 & 100.00 \\
\hline & Stylophora pistillata & 0.00 & 3.32 & 22.43 & 16.61 & 13.29 & 6.81 & 4.98 & 0.00 & 32.56 & 0.00 & 100.00 \\
\hline
\end{tabular}

species identified is significantly high compared to the total number in Nusalaut islands waters (123 species, Souhoka 2007), Marabatuan and Matasirih South Borneo (98 species, Munasik and Siringoringo 2011) and in Bunaken island waters (67 species, Kambey 2014). In contrast, the number of this species found in this study was smaller than the number of species found in Tobelo, North Halmahera (146 species,Souhoka 2012), in Tanjung Merah waters (165 species, Souhoka 2007) and in Lembata, East Nusa Tenggara (313 species, Abrar et al. 2012). The high number of hard coral species in Dampier Strait waters show that this location has quite a high variety of hard coral $30,92 \%$ of the total number of hard coral species that are found in Raja Ampat Regency waters (456 species, Veron 2002). The high number of hard coral species in Dampier Strait may be facilitated by the good condition of waters, with a range of salinity of $27.00-32.67 \%$, a temperature range of $25.00-31,20^{\circ} \mathrm{C}$ and range of $\mathrm{pH}$ of 7.34-7.39 (in situ measurement). Sukarno et al. (1981) stated that the growth of hard coral is generally possible in a range of salinity (25-40\%o.) The ideal temperature for the growth of hard coral ranges from $25-29^{\circ} \mathrm{C}$ in shallow tropical waters (Salm and Clark 1989).

\section{Benthic components of coral reefs}

The assessment of hard coral and the other of a benthic components in $3 \mathrm{~m}$ depth (Table 3) has shown that the highest hard coral was found in Station $5(86.83 \%)$ and the lowest in Station 8 (44.17\%.). The value of percentage live hard coral in $3 \mathrm{~m}$ depth consists of categories moderate to very good by Gomez and Alcala (1978) i.e 0-24.9\% (bad), 25-49.9\% (moderate), 50-74.9\% (good) and 75-100\% (very good). The highest hard coral cover in $6 \mathrm{~m}$ depth was found in Station $3(87.40 \%)$ and the lowest in station $2(51.17 \%)$. The average hard coral cover in two depth was found to range from $62.89 \%$ ( $6 \mathrm{~m}$ depth) to $65.69 \%$ (3m depth). This indicated that theses sites were in overall good condition. The average hard coral percentage cover in 3 and $6 \mathrm{~m}$ depth $(64.24 \%)$ also implied that the hard coral in Dampier Strait waters is in good condition. This value is significantly higher than other sites in Indonesia such as the Tegal and Sidodadi islands (49.87\%) (Hartoni et al. 2012) and Tagulandang, North Sulawesi (46.21\%) (Souhoka 2014).

A comparison of the hard coral percentage cover (Figure 2) shows that there was some variation between the research stations at 3 and $6 \mathrm{~m}$ depth. The value of category of bad condition ( $0-24.9 \%$ cover) was not found at any site. The moderate category $(25-49.9 \%$ cover) was found at $3 \mathrm{~m}$ depth (2 research stations), good condition (50-74.9\% cover) was found at 4 research stations in $3 \mathrm{~m}$ depth and 8 research stations in $6 \mathrm{~m}$ depth and very good condition (75$100 \%$ cover) was found in 4 stations ( $3 \mathrm{~m}$ depth) and 2 stations (in $6 \mathrm{~m}$ depth). Generally, the hard coral condition in $3 \mathrm{~m}$ depth had better cover than in $6 \mathrm{~m}$ depth. The high value of coral cover at $3 \mathrm{~m}$ depth may be because sunlight penetration was good enough so that the photosynthesis done by hard coral (zooxanthellae) would be continued. It is as stated by Wells (1956), that hard coral in the waters depended on the condition of around the waters i.e. sunlight, salinity, temperature, the movement of current, substrate and the brightness of water. The hard coral should not be limited horizontally but may be limited vertically by the depth of waters (Suharsono 2010). 


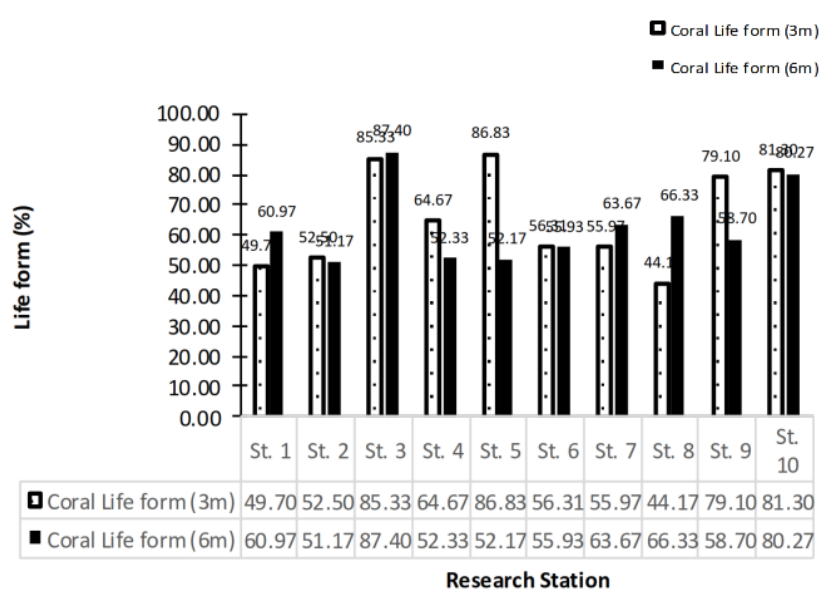

Figure 2. The percentage of hard coral life form in $3 \mathrm{~m}$ and $6 \mathrm{~m}$ in Dampier Strait marine protected area research stations, 2016

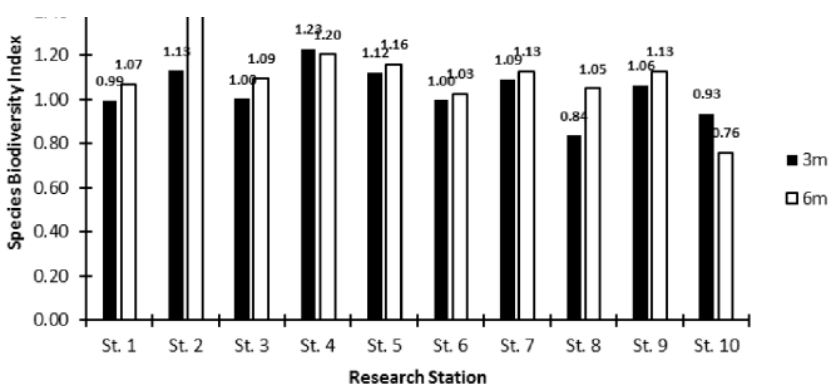

Figure 3. The value of species biodiversity index $(\mathrm{H})$ in $3 \mathrm{~m}$ and 6m depth, Dampier Strait research station, 2016

\section{Species diversity index}

The result of species diversity $(\mathrm{H})$ analysis showed that the highest diversity ( $3 \mathrm{~m}$ depth) was found in station 4 (1.23) and the lowest in station 8 (0.84). In $6 \mathrm{~m}$ depth, the highest value of species diversity index $(\mathrm{H})$ was found in station 2 (1.39) and the lowest in station $10(0.76)$ (Table $5)$. The high value of species diversity index $(\mathrm{H})$ iimplies that the community of biota was highly varied and was not dominated by one or two taxa (Romimohtarto and Juwana 1999). The comparison of species biodiversity index values between 3 and $6 \mathrm{~m}$ depth (Figure 3) showed that diversity was highest in $6 \mathrm{~m}$ depth, and the was found in 8 of the 10 stations. In contrast, a lower value of diversity was found in $6 \mathrm{~m}$ depth at station 4 (1.20) and station 10 (0.76).

The value of hard coral species diversity index $(\mathrm{H})$ in Dampier strait (Figure 3) in 3 and $6 \mathrm{~m}$ depth, generally showed that hard coral around of the waters had categories of productive and very productive (0.76-1.39) based on the criteria by Stodart and Johnson in Manuputty (1990) i.e 0.00-0.25 (no productive), 0.25-0.50 (a little productive), 0.50-0.75 (moderate productive), 0.75-1.00 (productive) and more than 1.00 (very productive). Alternatively, the value of species biodiversity (diversity index) found in the Dampier strait showed that the status of hard coral species biodiversity is in moderate condition, based on the criteria's
Krebs (1972) i.e. $0<\mathrm{H} \leq 1$ : low, $1<\mathrm{H} \leq 2$ : moderate and $\mathrm{H}>2$ : high (stable).

The value of species biodiversity was very high at station 2 (6 $\mathrm{m}$ depth), showing that hard coral in this location consists of a great variety of species. In contrast, the hard coral community in station 10 (6 m depth) consists of a relatively low species variety. The highest hard coral species especially was found from genus Acropora (19 species). Manuputty (1990) stated that hard coral from genera Acropora sp had a very small polyp and it is difficult to polyp to refresh by itself from any particle in coral's body. Consequently, these species need a strong current and waves as found in the Dampier straits to survive.

The value of hard coral species evenness (j) in Dampier (Table 5) in both depths ranged from 0.73 (station 10) $6 \mathrm{~m}$ depth to 0.94 (station 4) $6 \mathrm{~m}$ depth. The value of evenness index is usually used to assess the distribution pattern of biota i.e. evenly distributed or unevenly distributed (Romimohtarto dan Juwana 1999). The high value of evenness index in station 4 shown that hard corals are distributed in a homogeneous way and there are no dominant colonies (patches). Meanwhile at the station 10, hard corals distributed less homogeneously than at any other station, especially because of the species Seriatophora hystrix and Echinopora lamellosa that were found a lot at this station. According to Suharsono (2010), the coral reef species Seriatophora hystrix and Echinopora lamellosa are generally common in around Indonesian waters and scatter around the reef flat. The value of evenness index found in 10 Dampier strait research stations indicated that hard coral communities are in a stable condition, based on criteria by Krebs (1972) $0<\mathrm{E} \leq 0.50$ : stressed community, $0.50<\mathrm{E} \leq 0.75$ : label community and $0.75<\mathrm{E} \leq 1.50$ : stable community.

Species dominance index (D) revealed the presence and absence of species dominance throughout the sampling area. The result showed that the dominance index ranged from 0.05 (station 2) to 0.25 (station 10). The high value of species dominance in station 10 showed that dominance of hard coral species was greater here than at other sites. The highest dominance of hard coral species was found Seriatophora hystrix and Echinopora lamellosa and was distributed in quite wide areas. Veron (1986) found these species were also common from the Great Barrier Reef, Coral Sea, Moreton Bay, and Lord Howe Island in the east to west coast of Australia.

\section{The length of coral reef colony}

The length of a hard coral colony in $3 \mathrm{~m}$ and $6 \mathrm{~m}$ (Table 6 and Table 7) showed that the species Acropora sp had the total length of $4194 \mathrm{~cm}$. The highest length was found in station $9(1258 \mathrm{~cm})$ (Table 8$)$ and was found in every research station (10 stations) in Dampier strait waters (Table 6). Acropora sp has found living in quite wide areas. Suharsono (2010) stated that hard coral from the genus Acropora was found in almost in every Indonesian water, and Veron (1986) found this coral in Madagascar, the east island of Marshall, Great Barrier Reef, south of Coral Sea to the east island of Lord Howe and Scott Reef in the west 
coast of Australia waters. Another hard coral species found to be quite long Porites lutea $(1804 \mathrm{~cm})$ in $3 \mathrm{~m}$ depth and this species found dominance in station $1(439 \mathrm{~cm})$ (Table 8). Porites lutea was formed a massive coral, where the part of a surface colony impressed smooth with the form of corallite uniform and distributed across Indonesian water and found very generally on reef flat (Suharsono 2010). Porites have well adapted to changes of environment in the waters. According to Tomascik and Sander (1987) they, found the hard coral species Porites lived in waters with eutrophication.

In conclusions, the number of hard coral in Dampier Strait waters, Raja Ampat regency consists of 141 species that represented 16 families. The average hard coral condition in categories good with the percentage of life form in range $62.89 \%$ ( $6 \mathrm{~m}$ depth) to $65.69 \%$ (3m depth). The highest percentage of hard coral life form was found in station 3 (6m depth) in $87.40 \%$ and the lowest was found in station 8 (3m depth) in $44.17 \%$. The biodiversity of hard coral is productive to very productive with moderate stability growth. The total length of a hard coral growth area in 10 Dampier Strait research station is $39.35 \%(3 \mathrm{~m}$ depth) and $37.74 \%$ (6m depth) from the total length of the transect.

\section{ACKNOWLEDGEMENTS}

We like to convey our gratitude to the Director of Coastal and Marine Resources Management Program, Graduate School, Bogor Agricultural University, Indonesia who has given us a chance to author research into Dampier Strait marine protected area with respect to the completion of the writing of a dissertation (S3). We like to express our gratitude and appreciation to the provincial government of West Papua, the regency government of Raja Ampat and University of Papua (UNIPA), Manokwari, Indonesia as well as all parties for contributions given during the collection of data in the research stations.

\section{REFERENCES}

Abrar M, Bachtiar I, Budiyanto A. 2012. The structure of community and coral (Scleractinia) diseases in Lembata waters, East Nusa Tenggara. Mar Sci 17 (2): 109-118.

Allen GR, Erdmann MV. 2009. Reef fishes of the Bird's Head Peninsula, West Papua, Indonesia. Check list 5: 587-628

Boli P, Yulianda F, Damar A, Soedharma D, Kinseng R. 2014. Benefits of Sasi for Conservation of Marine Resources in Raja Ampat, Papua. JMHT 20 (2): 131-139. DOI: 10.7226/jtfm.20.2.131

English S, Wilkinson C, Baker V. 1997. Survey manual for tropical marine resources. Second edition. Aust Inst Mar Sci Townsville: 390.

Larsen SN, Leisher C, Mangubhai S, Muljadi A, Tapilau R. 2011. Laporan Penilaian Desa Pesisir di Kabupaten Raja Ampat, Papua Barat, Indonesia. The Nature Conservancy, Sorong.

Gomez ED, Alcala AC. 1978. Status of Philiphina coral reef. The project, Int Symp Biogeogr Evol S Hem Auckland New Zealand, 17-20 July 1978. 2: 663-669.

Hartoni, Damar A, Wardiatno Y. 2012. The condition of coral reef in Tegal and Sidodadi Islands waters. District of Padang Cermin, Pesawaran Regency, the Province of Lampung. Maspari J 4 (1): 4657. [Indonesian]
Huang Z, Mao L. 2012. The living species and their illustrations in China's Seas (Part II). An illustrated guide to species in China's Seas. China Ocean Press, Beijing.

Huffard CL, Erdmann MV, Gunawan T. 2009. Defining Geographic Priorities for Marine Biodiversity Conservation in Indonesia. Coral Triangle Support Program, Jakarta. [Indonesian]

Kambey AD. 2014. Conditions of Coral Reef in Bunaken Island North Sulawesi Province. Jurnal Ilmiah Platax 2 (1): 19-24. [Indonesian]

Koh EGL, Chou LM. 1989. The mushroom corals of Singapore Departement of Zoology, National University of Singapore, Singapore.

Krebs CJ. 1972. Ecology. The Experimental Analysis of Distribution and Abundance. Harper and Row Publisher, New York, USA.

Manuputty AE. 1990. The distribution of biodiversity and stony coral composition in Kabil waters. Soemodihardjo, S.S. Birowo dan K. Romimohtarto (Eds). Batam Island. The Research and Development Center of Oceanography-Indonesian Institute of Sciences, Jakarta. [Indonesian]

Mangubhai S, Erdmann MV, Wilson JR, Huffard CL, Ballamu F, Hidayat NI, Hitipeuw C, Lazuardi ME, Muhajir, Pada D, Purba G, Rotinsulu C, Rumetna L, Sumolang K, Wen W. 2012. Papuan Bird's Head Seascape: Emerging Threats and Challenges in The Global Center of Marine Biodiversity. Mar Pollut Bull 64 (11): 2279-2295.

McWilliam M, Hoogenboom MO, Baird AH, Kuo C. 2018. Biogeographical disparity in the functional diversity and redundancy of corals. Proc Natl Acad Sci 115 (12): 201716643. DOI: $10.1073 /$ pnas.1716643115.

Moll H, Moka W. 1986. Indonesia-Deutch Snellius II Expedition. Compedium 4. Guide to Indonesia Reef Corals. Rijksmuseum Natuurlijke Historie Leiden.

Munasik, Siringoringo R. 2011. The structure of hard coral community (Scleractinia) in Marabatuan and Matasirih islands waters, South of Borneo. Mar Sci 16 (1): 49-58.

Mustaghfirin, Urbinas MP, Erdman MV, Mangubhai S, Fox M, Khazali M, Rumetna L, Nebore A, Thebu K, Setyawan D, Mongdong M, Djunaidi A, Wamafma K, Maturbongs J, Purwanto, Muhajir, Hidayat NI, Sabonnama S. 2012. The second books of report: The management plant of Small Islands Park in Raja Ampat Areas: The data and analize. The implementation unit of Small Islands Park Raja Ampat Areas. Departement of Marine and Fisheries, The Government of Raja Ampat, West Papua Province.

Nybakken JW, Bertness MD. 2004. Marine Biology: An Ecological Approach, 6th ed.. Benjamin Cummings, London.

Odum EP. 1971. Fundamentals of ecology. W. E. Saunders, Philadelphia: 574 pp.

Romimohtarto K, Juwana S. 1999. Marine Biology. The Science of Marine Biota. Research and Development Center of OceanographyIndonesian Institute of Sciences, Jakarta. [Indonesian]

Salm RV, Clark JR. 1989. Marine and coastal protected areas. A guide for planner and managers. International Union for the conservation of nature and natural resources. Gland, Switzerland.

Souhoka J. 2007. The distribution and condition of stony coral in Tanjung Merah Bitung waters, North Sulawesi. Oceanol Limnol 33 (3): 393411. [Indonesian]

Souhoka J. 2012. The condition of stony coral in Mantihage islands waters North Minahasa Regency, The Province of North Sulawesi. Biota Sci J Biodivers 17 (3): 153-164.

Souhoka J. 2014. The structure of stony coral community (Scleractinia) in Tagulandang waters, North Sulawesi. Oceanol Limnol 40 (2): 189202. [Indonesian]

Suharsono. 2010. The biodiversity of coral reef in Indonesia. Indonesian Institute of Sciences - Coremap Program, Jakarta. [Indonesian]

Sukarno, Hutomo M, Moosa MK, Darsono P. 1981. The Coral Reef in Indonesia. The resources, problem, and management. The research project of natural resources potential in Indonesia. National Oceanology Institution, Indonesian Institute of Sciences, Jakarta. [Indonesian]

Sumadhiharga OK, Djamali A, Badrudin M. 2006. Keanekaragaman Jenis Ikan Karang di Perairan Belitung Barat, Kepulauan Bangka Belitung. Ilmu Kelautan 11 (4):201-209. [Indonesian]

Tomascik T, Sander F. 1987. Effects of eutrophication on reef corals. II. Structure of scleractinian coral communities on fringing reefs, Barbados, West Indies. Mar Biol 94: 53-75.

Veron JN. 1986. Coral of Australian and the Indo-Pacific. University of Hawaii Press, Honolulu. 
Veron JN, Hoegh-Guldberg O, Lenton T, Loug J, Obura D, Pearce-Kelly P, Sheppard C, Spalding M, Stafford-Smith M, Rogers A. 2009. The coral reef crisis: The critical importance of $<350 \mathrm{ppm}$ CO2. Mar Pollut Bull 58: 1428-1436.
Wallace CC, Turak E, DeVantier L. 2011. Novel characters in a conservative coral genus: three new species of Astreopora (Scleractinia: Acroporidae) from West Papua. J Natl Hist 45: 19051924 